\title{
Effect of strain on surface diffusion in semiconductor heteroepitaxy
}

\author{
Evgeni Penev, Peter Kratzer, and Matthias Scheffler \\ Fritz-Haber-Institut der Max-Planck-Gesellschaft, Faradayweg 4-6, 14195 Berlin-Dahlem, Germany
}

(Submitted 25 January 2001)

\begin{abstract}
We present a first-principles analysis of the strain renormalization of the cation diffusivity on the $\operatorname{GaAs}(001)$ surface. For the example of In/GaAs(001)-c(4 ×4) it is shown that the binding of In is increased when the substrate lattice is expanded. The diffusion barrier $\Delta E(\varepsilon)$ has a non-monotonic strain dependence with a maximum at compressive strain values $(\varepsilon<0)$, while being a decreasing function for any tensile strain $(\varepsilon>0)$ studied. We discuss the consequences of spatial variations of both the binding energy and the diffusion barrier of an adatom caused by the strain field around a heteroepitaxial island. For a simplified geometry, we evaluate the speed of growth of two coherently strained islands on the $\operatorname{GaAs}(001)$ surface and identify a growth regime where island sizes tend to equalize during growth due to the strain dependence of surface diffusion.
\end{abstract}

\section{INTRODUCTION}

In recent years, the heteroepitaxial growth of latticemismatched semiconductor systems has attracted substantial interest. For a number of systems (e.g. Ge/Si, InAs/GaAs), it could be shown that under appropriate experimental conditions the deposited material forms elastically strained, dislefation-free (coherent) threedimensional (3D) islands. ten show a rather narrow size distribution, in particular in the higher layers of a stacked 3D array of islands obtained from repeated deposition of heteroepitaxial films separated by spacer layers. This feature is essential for the usefulness of these nanostructures as quantum dots (QDs) and for their envisaged application in future optoelectronic devices 3 Considerable theoretical efforts have been made in order to rationalize the observed regularities in island sizes and ordering. Some approaches have attempted to describe the islands as equilibrium structures 1 As an alternative explanation, the role of $k i$ netics for the growth of heteroepitaxial islands has been emphasized. 0 It is possible that intrinsic features of the kinetics of the growth process give rise to regular structures. For instance, self-limiting effects in strained island growth could result in a preferred island size, either due to a limitation in material supply 10 or due to nucle ation barriers in the growth of the islands' side facets. 11 This perspective motivated an intense theoretical effort towards better understanding the underlying microscopic processes in molecular beam epitaxy (MBE), e.g. deposition, diffusion and nucleation. First-principles calculations have already been applied to study different aspects of cation diffusion on unstrained compound semiconductor surfaces.12. 13 Up to now, however, the impact of strain on the diffusion process still remains elusive, although attempts to make its effect clearer date back to the last decade.14

We illustrate the importance of strain for typical heteroepitaxial systems (e.g. Ge/Si, InAs/GaAs) for two situations: First, during growth of free-standing het- eroepitaxial islands, the islands themselves are under compressive strain, whereas the substrate beneath the island is expanded. As a consequence of this expansion, the substrate surface around an island is under compressive strain (see, e.g. Ref. 15). Supply of further material to the growing island is governed by diffusion through this ring-shaped area of compressive strain. A suppressive effect of strain on diffusion could slow down the growth of larger islands. Secondly, in the growth of three-dimensional (3D) stacked arrays of QDs, the buried islands act as stressors causing tensile strain on the capping layer in the regions above them.16.17 Again, this may affect the growth kinetics of the next layer of islands to be formed on the capping layer. Thus, in different stages of growth of a nanostructure, different regimes of strain may come into focus.

From the limited published data about strain effects on diffusion, it appears surprising that compressive surface strain could lead to a self-limiting effect on the island growth. First-principles calculations for diffusion on close-packed metal surfaces, in particular $\mathrm{Ag} / \mathrm{Ag}(111)$, Ref. 18, have demonstrated that compressive strain increases the adatom diffusivity by reducing the diffusion barrier. Schroeder and Wol 19 have extended this finding to diffusion on (001) surfaces of simple cubic, fcc, and bcc lattices. Recent molecular dynamics (MD) simulations using empirical potentials showed the same trend for Ga, In, and As adatom_liffusion on a $(2 \times 1)$-reconstructed GaAs(001) surface.20 These results also comply with an earlier idy of Ga kinetics on the strained $\mathrm{GaAs}(001)$ surface.14 A different strain dependence of diffusion was found, however, for Si adatom 2122 and dimer23 diffusion on the $\mathrm{Si}(001)$ surface, where tensile strain leads to an overall decrease in the diffusion barriers. Yet, the majorities of the theoretical studies on semiconductor systems provide only scarce quantitative information about the influence of strain on the diffusion process.

The aim of this article is two-fold: First, we report the results of density-functional theory (DFT) calculations for the tracer diffusion 25 of a single In adatom 
on a GaAs surfaces. In particular, we investigate the strain dependence of diffusion in order to clarify the issues raised above concerning heteroepitaxy of a strained system. This problem can be viewed as a $2 \mathrm{D}$ analeg to the effect of pressure on diffusion in bulk materials.24 In a second part, we discuss the impact of these findings for growth for two typical situations, nucleation on a strained capping layer for low In concentrations, and diffusionlimited growth of quasi-one-dimensional islands. While these topics have been discussed in the literature in the context of thermo-chemical diffusion,26,27,10,16 our focus will be on a kinetic description inspired by the results of our atomistic calculations.

For a systematic first-principles investigation of the effect of strain on the diffusivity of an In adatom, we decided to use the $\mathrm{GaAs}(001)-c(4 \times 4)$ surface 29 as a specific example. On top of a complete As surface layer, the $c(4 \times 4)$ reconstruction has rows of As dimers running in the $[\overline{1} 10]$ direction, with units of three As dimers interrupted by a dimer vacancy (see Fig. 1 below). We have chosen this reconstruction because it forms the substrate for the initial stages of InAs deposition for temperatures $T \lesssim 500^{\circ} \mathrm{C}$ (see Ref. 30). For very arsenic-rich growth conditions, In deposition is expected to lead to direct formation of 3D InAs islands, as has been shown by previous calculations. 31 Thus, we can use this system to study both the diffusion of the first In atoms on a strained substrate (e.g. a capping layer with buried islands), as well as diffusion in the vicinity of an InAs island on the surface. We note, however, that the commonly used growth conditions to fabricate quantum dots involve formation of a InAs wetting layer with reconstructions different from the $c(4 \times 4)$. Diffusion of In on this wetting layer will be addressed in a future publication.

In Sec. II we outline the underlying computational method. The mapping of the potential energy surface (PES) for In diffusion on the unstrained $\operatorname{GaAs}(001)$ $c(4 \times 4)$ surface is presented in Sec. III A. In Sec. III B we discuss in great details the In adatom interaction with the surface As dimers. The effect of strain is then addressed in Sec. III Q. All microscopic results are critically examined in Sec. [V] in order to assess possible morphological consequences for the growth of strained InAs islands on GaAs. Finally, a summary and discussion of the results is presented in Sec. $\mathrm{V}$.

\section{COMPUTATIONAL DETAILS}

DFT calculations have proven to be an efficient tool to explore the elementary processes of crystal growth (see for example Ruggerone Ratsch and Scheffler 32 ). In the setting employed here 33 the substrate is modeled by a slab, representing the topmost seven atomic layers of the $\operatorname{GaAs}(001)-c(4 \times 4)$ surface, the bottom layer of which was passivated by pseudo-hydrogen atoms. A plane-wave basis set with $E_{\text {cut }}=10$ Ry energy cut- off was 1 ised in conjunction with $a b$ initio pseudopotentials 34 and the Perderv-Burke-Ernzerhof generalized gradient approximation 35 to the exchange and correlation was employed throughout this study. The integration over the surface Brillouin zone (SBZ) was performed using a Monckhorst-Pack set with two special k-points, $\left[\left(\frac{1}{2}, 0,0\right),\left(0, \frac{1}{2}, 0\right)\right]$, equivalent to $64 \mathbf{k}$-points in the irreducible part of the $1 \times 1$ SBZ. Thus, the $\mathbf{k}$-mesh conforms with the one previously used for the $\beta 2(2 \times 4)$ reconstruction.12

It is common practice, when addressing adatom diffusion, to map out the relevant potential energy surface(s) that contains complete information abput the diffusion process. Kley, Ruggerone and Scheffler 12 have recently argued that the adatom-dimer interaction on the $\operatorname{GaAs}(001)$ surface is a crucial factor for the proper determination of the PES. In order to set the stage for introducing strain into the problem, we have scanned the potential-energy landscape seen by the In adatom, by relaxing the latter along the surface normal, placing it laterally over a set of equidistant grid points in the (001) plane and allowing the topmost six slab layers to freely relax. As a reference for calculating the binding energy of the adatom we have used the sum of the total energy of the (properly strained) baresurface and the energy of a free, spin-polarized In atom 36 Geometries were considered converged when all residual forces were smaller than $0.025 \mathrm{eV} / \AA$.

The adatom-dimer interaction poses a multidimensional problem, since not only the adatom itself, but also all degrees of freedom of the surface atoms are involved in this processes. Therefore, even a full relaxation starting from an adatom above the surface may only lead to a local minimum, while other minima may exist that can only be reached from different starting configurations. Test calculations showed that the adatomsurface distance and the As-As distance in the dimer are most important, and the 2D configurational space defined by these coordinates is suitable to image the In-surface dimer interaction. Towards this end a special constrained relaxation was carried out allowing the In adatom and the central As dimer beneath it to be moved as a rigid unit (see the inset in Fig. 3 below). The relative position of these three atoms defines a point in a $2 \mathrm{D}$ slice through the corresponding multidimensional energy hypersurface. Performing the constrained relaxation, we succeeded in mapping out the 2D PES governing the In-surface interaction in a point-by-point fashion. Further details will be given in Sec. III B.

The strain field at a step or island edge varies slowly on the scale of the lattice constant. Therefore we can treat the inhomogeneously strained surface by performing DFT calculations with a locally adjusted lattice parameter. Accordingly, to investigate the influence of strain on surface diffusion, the lateral lattice constant $a$ was uniformly changed in the range of $\pm 8 \%$ around its value $a_{0}$ calculated for the unstrained material, thus defining the isotropic surface strain tensor $\varepsilon_{\alpha \beta}=\varepsilon \delta_{\alpha \beta}$, 

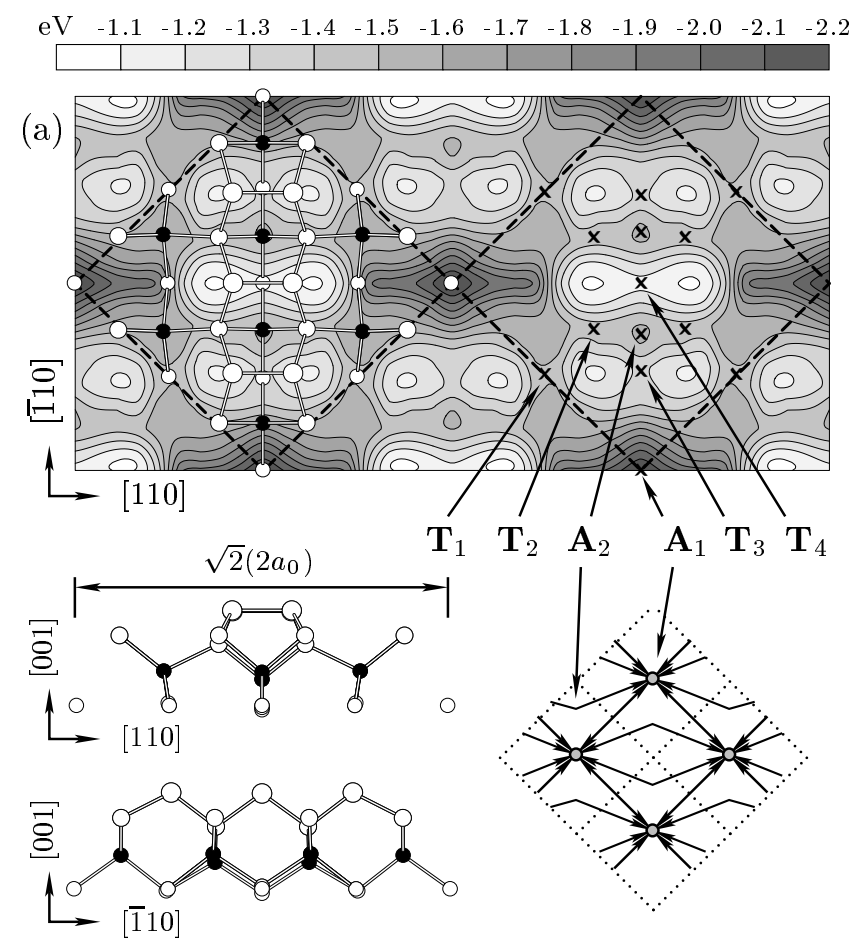

(b)

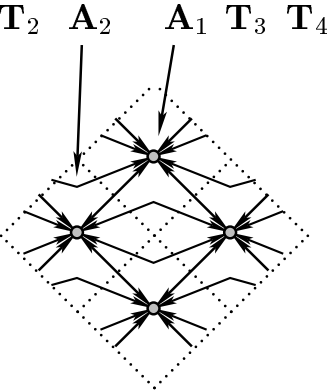

(c)

FIG. 1. (a) Potential-energy landscape for an In adatom on the $\operatorname{GaAs}(001)-c(4 \times 4)$ surface. The adatom is relaxed from $2 \AA$ above the surface. Contour-line spacing is $0.1 \mathrm{eV}$; atomic positions in the clean surface unit cell are indicated for atoms in the upper four layers (As: empty circles; Ga: filled circles), side views shown in panel (b), where $a_{0}$ is the bulk GaAs lattice constant. The dashed squares show the surface unit cell. (c) Sketch of the 2D network of sites used in the random walk formalism (four unit cells are depicted).

with $\varepsilon=a / a_{0}-1, \delta_{\alpha \beta}$ being the Kronecker delta, relaxing again the system as already explained and recording the In binding energy for the relevant sites on the PES. It is important to note that any change of the super-cell volume $V$ is accompanied by a change in the quality of the plane-wave basis set. To account for this effect we have corrected the calculated total energy of the supercell $E_{\text {tot }}\left(E_{\text {cut }}, V\right.$ according to the scaling hypothesis by Rignanese et al..37

\section{INDIUM DIFFUSION ON GaAs(001)-c(4 ×4)}

\section{A. Potential energy surface}

The mapping procedure resulted in the PES shown in Fig. 1 and the binding energies of the In adatom at the adsorption sites $\left(\mathbf{A}_{i}\right)$ and saddle points $\left(\mathbf{T}_{k}\right)$ are given in Table If. The In adatom, like Ga/GaAs(001)-c(4 × 4) (cf. Ref. 13 and Table I), preferentially adsorbs at the four-fold coordinated hollow site $\mathbf{A}_{1}$ (the missing dimer

TABLE I. Binding energy $E_{b}(\mathrm{eV})$ of an In adatom at the sites on the $\operatorname{GaAs}(001)-c(4 \times 4)$ surface, denoted in Fig. 1. For comparison, $E_{b}$ of a $\mathrm{Ga}$ adatom at the same sites are read off the corresponding potential-energy map calculated by LePage et al. in Ref. 13 using the local-density approximation.

\begin{tabular}{lcccccc}
\hline \hline & \multicolumn{6}{c}{ Site } \\
\cline { 2 - 7 } & $\mathbf{A}_{1}$ & $\mathbf{A}_{2}$ & $\mathbf{T}_{1}$ & $\mathbf{T}_{2}$ & $\mathbf{T}_{3}$ & $\mathbf{T}_{4}$ \\
\hline In & -2.21 & -1.54 & -1.56 & -1.44 & -1.27 & -1.17 \\
Ga & -3.04 & -2.20 & -2.54 & -2.10 & -2.00 & -1.90 \\
\hline \hline
\end{tabular}

position), where it interacts with the dangling bonds of the second layer As atoms. Two other very shallow minima $\mathbf{A}_{2}$ are located in between the center and the two edge dimers. Jumps between the adsorption sites occur through four symmetry-inequivalent saddle points $\mathbf{T}_{k}$, with $\mathbf{T}_{1}$ being lowest in energy.

Within transition-state theory 38 the hopping rate between sites $\mathbf{A}_{i}$ and $\mathbf{A}_{f}$, crossing saddle point $\mathbf{T}_{k}$, is given by the ratio of the partition functions of the system with the adatom at the equilibrium site, $Z_{i} \equiv Z\left(\mathbf{A}_{i}\right)$, and at the saddle point, $Z_{k} \equiv Z\left(\mathbf{T}_{k}\right)$,

$$
\Gamma_{f i}=\frac{1}{2 \pi} \frac{k_{\mathrm{B}} T}{\hbar} \frac{Z_{k}}{Z_{i}}
$$

where $\hbar$ is the Planck's constant. In the conventional case of an elastically relaxed surface the Helmholtz free energy $F=-k_{\mathrm{B}} T \ln Z$ is the proper thermodynamic potential. Eq. (1) can be cast into the well known Arrhenius form

$$
\Gamma_{f i}=\Gamma_{f i}^{(0)} \exp \left\{-\left[E\left(\mathbf{T}_{k}\right)-E\left(\mathbf{A}_{i}\right)\right] / k_{\mathrm{B}} T\right\}
$$

where $E$ is the (static) total energy of the system, read off the PES in Fig. 1. The prefactor $\Gamma_{f i}^{(0)}$ has the form,

$$
\Gamma_{f i}^{(0)}=\frac{1}{2 \pi} \frac{k_{\mathrm{B}} T}{\hbar} \exp \left(-\Delta U_{\mathrm{vib}} / k_{\mathrm{B}} T+\Delta S_{\mathrm{vib}} / k_{\mathrm{B}}\right),
$$

where $\Delta U_{\text {vib }}$ and $\Delta S_{\text {vib }}$ are, respectively, the associated changes in the vibrational energy and entropy. $\Gamma_{f i}^{(0)}$ is temperature-independent within the classical harmonic approximation.

In a simplified picture, the In adatom migrates by a random walk on a $2 \mathrm{D}$ square lattice defined by the $\mathbf{A}_{1}$ sites, Fig. 1 (c). However, we account for hops between them via both $\mathbf{T}_{1}$ and $\mathbf{T}_{2}-\mathbf{A}_{2}-\mathbf{T}_{2}$ with rates $\Gamma_{11}$ and $\tilde{\Gamma}_{11}$, respectively. Indeed, once the In adatom has reached the $\mathbf{A}_{2}$ site it needs to overcome a barrier $E\left(\mathbf{T}_{2}\right)-E\left(\mathbf{A}_{2}\right)$ of only $0.1 \mathrm{eV}$ in order to move towards a neighboring $\mathbf{A}_{1}$ site. As $E\left(\mathbf{T}_{2}\right)-E\left(\mathbf{A}_{2}\right) \lesssim 2 k_{\mathrm{B}} T$ for typical growth temperatures, the adatom is unlikely to equilibrate at the shallow well $\mathbf{A}_{2}$ before it escapes. Thus it is justified to use a single rate $\tilde{\Gamma}_{11}$ for the whole path $\mathbf{T}_{2}-\mathbf{A}_{2}-\mathbf{T}_{2}$.

Effective diffusion coefficients can now be extracted by applying the continuous-time random walk (CTRW) formalism.12 39 Thus, it is easily worked out that the 
diffusion tensor in Cartesian coordinates $(x \|$ [110], $y \|[\overline{1} 10])$ reads

$$
D_{\alpha \beta}=\left(\begin{array}{cc}
D_{[110]} & 0 \\
0 & D_{[\overline{1} 10]}
\end{array}\right)=4 a_{0}^{2}\left(\begin{array}{cc}
\Gamma_{11}+4 \tilde{\Gamma}_{11} & 0 \\
0 & \Gamma_{11}
\end{array}\right) .
$$

The factor 4 in front of $\tilde{\Gamma}_{11}$ is partly due to the fact that there exist two equivalent $\mathbf{T}_{2}-\mathbf{A}_{2}-\mathbf{T}_{2}$ paths across the block of three dimers. Another factor two enters because the In adatom travels in [110] direction $\sqrt{2}$ times longer distance to reach a neighboring $\mathbf{A}_{1}$ site than along the path crossing the saddle $\mathbf{T}_{1}$. Eq. (伍) thus implies that an isolated In adatom migrates slightly faster in [110] direction, across the dimer rows, than along the dimer rows in [110] direction, with anisotropy ratio $D_{[110]} / D_{[\overline{1} 10]}=1+4 \tilde{\Gamma}_{11} / \Gamma_{11}$. The related diffusion barriers, entering the rates $\Gamma_{11}$, and $\tilde{\Gamma}_{11}$ are $\Delta E=0.65 \mathrm{eV}$ and $\Delta \tilde{E} \simeq 0.8 \mathrm{eV}$ (cf. Fig. 自 (a), and Table更). One gets a rough estimate for the contribution of the $\mathbf{A}_{1} \stackrel{\mathbf{A}_{2}}{\longleftrightarrow} \mathbf{A}_{1}$ channel by assuming that $\Gamma_{11}^{(0)}$ and $\tilde{\Gamma}_{11}^{(0)}$ differ inessentially. Thus, e.g. at $T=450^{\circ} \mathrm{C}, D_{[110]} / D_{[\overline{11} 10]}$ would exceed unity by about $50 \%$. At sufficiently low temperatures, however, one should include $\mathbf{A}_{2}$ in the $2 \mathrm{D}$ network of sites and consider branching of the diffusion pathways towards neighboring $\mathbf{A}_{1}$ or $\mathbf{A}_{2}$ sites. Although an analytic result for $D_{\alpha \beta}$ can still be derived in this case within the CTRW formalism, the expressions are rather cumbersome and one has to seek for simplifications requiring knowledge of all $\Gamma_{f i}$ rates.

In comparison to the results by LePage et al. 13 (Table $\mathbb{\text { Ii) }}$ for $\mathrm{Ga} / \mathrm{GaAs}(001)-c(4 \times 4)$, In appears to diffuse on a less corrugated PES. Thus, for example, at the $\mathbf{A}_{1}$ site, being the most stable for both In and $\mathrm{Ga}$, the In adatom is by $\approx 0.8 \mathrm{eV}$ less bound than Ga. Furthermore, the $c(4 \times 4)$ PES provides two additional adsorption sites for Ga as compared to In: between the edge dimers ( $\mathbf{T}_{1}$ in Fig. 1) as well as in between $\mathbf{A}_{1}$ and the center dimer along [110] (cf. Fig. 2 of Ref. 13). At the $\mathbf{T}_{1}$ site, which is a stable adsorption site for Ga, the latter is by $1.0 \mathrm{eV}$ more strongly bound than In. These differences can be easily rationalized in terms of the differences in the cation-As bond strength in the corresponding binary compounds (GaAs, InAs) and the larger ionic radius $R_{\mathrm{In}}$ of indium: 40 we note however that part of the differences is to be attributed to the use of the local density approximation in Ref. 13. The cohesive energy per cation-As pair is lowest for InAs $\left(E_{\mathrm{coh}}^{\operatorname{InAs}}=6.20 \mathrm{eV}\right)$ as compared to $\operatorname{GaAs}\left(E_{\mathrm{coh}}^{\mathrm{GaAs}}=6.52 \mathrm{eV}\right)$ and $\mathrm{AlAs}\left(E_{\mathrm{coh}}^{\mathrm{AlAs}}=7.56 \mathrm{eV}\right)$, see e.g. Ref. 41. The barriers for diffusion of group-III cations on the GaAs surface follow the trend given by the binding energies, as has been also observed in a firstprinciples study of $\mathrm{G}$ a and $\mathrm{Al}$ diffusion on the $\mathrm{GaAs}(001)$ $\beta 2(2 \times 4)$ surface. 12

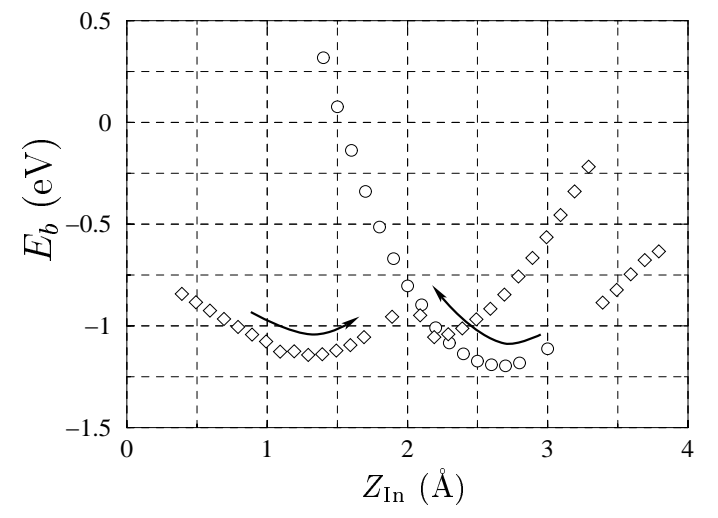

FIG. 2. Binding energy of an In adatom interacting with the center As dimer as a function of the $z$-coordinate of the adatom $Z_{\text {In }}$. The latter is measured from the $z$-coordinate of the center dimer for the bare surface. Arrows indicate the order in which the calculations were performed: adsorption (॰) and desorption $(\diamond)$.

\section{B. Interaction of Indium with As-As bonds}

Since the $c(4 \times 4)$ reconstruction represents a double layer of arsenic, of which the top As atoms form As dimers (Fig. 1), the incorporation of In into the cation sublattice requires the topmost arsenic layer to be eventually replaced by In atoms. One obvious way how this incorporation could occur is by splitting of the As-As bonds in a reaction with an In adatom. For an understanding of heteroepitaxy, it is therefore important to study such processes. Furthermore, Kley, Ruggerone and Scheffler 12 have pointed out for $\mathrm{Ga} / \mathrm{GaAs}(001)-\beta 2(2 \times 4)$ that the adatom interaction with the surface As dimers has important consequences for the cation diffusivity, since the broken As-As bond provides a very stable adsorption site for Ga. The underlying mechanism has been identified to be the replacement of the rather weak surface As-As dimer bond by stronger cation-As bonds, cf. Ref. 12. For a valid description of In diffusion by the PES shown in Fig. 1. we therefore have to check if reaction of In with the As-As bonds can lead to more stable binding sites for In than the minima of the PES.

We first sample $E_{b}$ as a function of the adatom height $Z_{\text {In }}$ above the $\mathbf{T}_{4}$ site, see Fig. 2. We perform a series of calculations for various values of $Z_{\text {In }}$, where in each calculation the adatom is kept fixed, while the substrate is allowed to freely relax. In subsequent calculations of the series, the geometry of the substrate atoms from the previous calculation is used as input. We find that the outcome of such a series of calculations depends on the initial geometry. While a set of data points modeling adsorption, starting from $Z_{\text {In }}=3 \AA$ above the closed dimer, shows an energy minimum at $Z_{\text {In }} \simeq 2.7 \AA$, a series of calculations for desorption, starting from an adatom incorporated in between the As dimer atoms at $Z_{\text {In }} \simeq 0.5 \AA$, finds a minimum at $Z_{\text {In }} \simeq 1.3 \AA$. Both minima have 


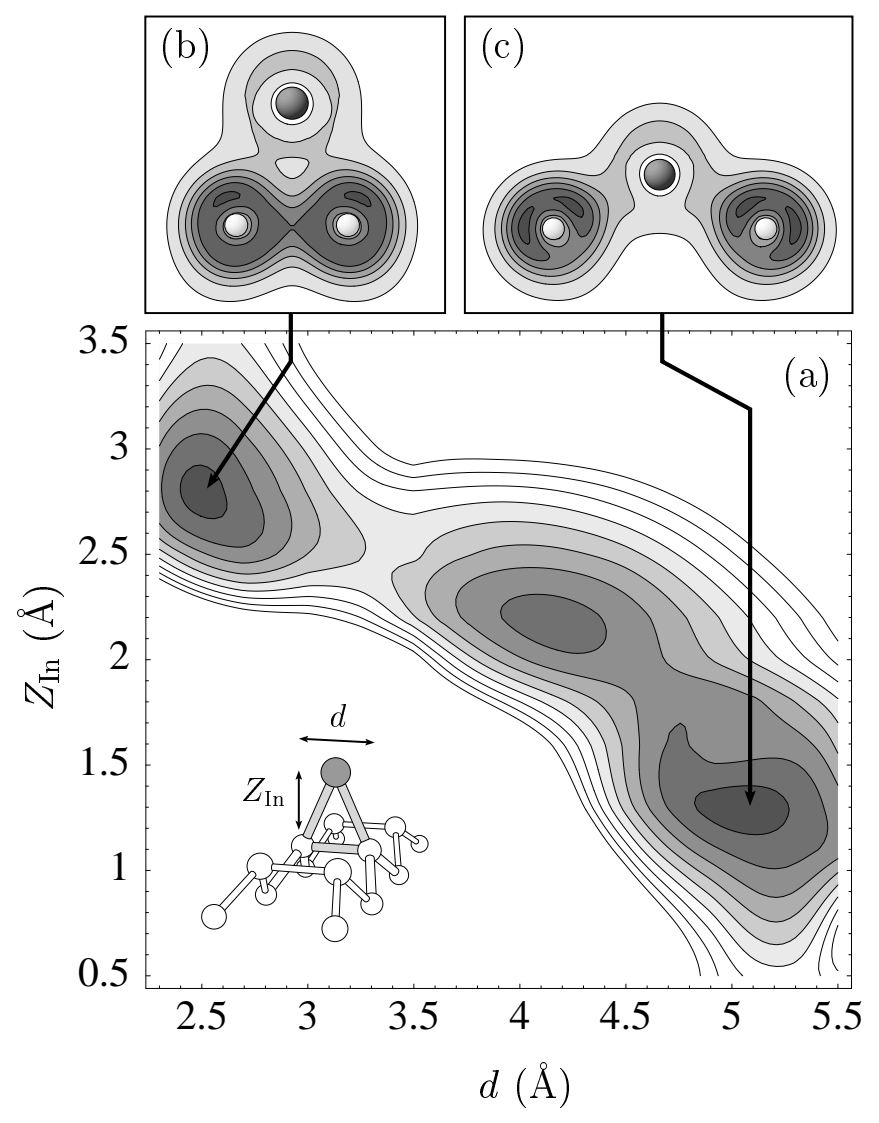

FIG. 3. (a) Binding energy of an In adatom interacting with the center As dimer as a function of the As-As distance $d$ and the In height above the midpoint of the dimer $Z_{\text {In }}$, as indicated in the inset. (b,c) Bonding configuration and the valence electron density in the plane containing the adatom and the dimer for the two deeper minima of $E_{b}$.

nearly the same depth, $E_{b} \simeq-1.2 \mathrm{eV}$. The corresponding bonding configurations and total valence electron densities are shown in Fig. 3 (b) and (c). The discontinuity and hysteresis in $E_{b}$ seen in Fig. 22 due to the dimer opening or closing indicates that the information gained from the $Z_{\text {In }}$ coordinate alone is insufficient for building up a complete picture of the adatom-dimer interaction.

Therefore we have subsequently imaged $E_{b}$ in a $2 \mathrm{D}$ configurational space including also the As-As distance $d$. In these calculations, both $d$ and the distance between the $z$-coordinate of the adatom and the As-As midpoint are held fixed while relaxing the remaining coordinates of the system. As a result, it becomes evident that the adatom, approaching the dimer, first goes through a minimum of $E_{b}$ with the dimer bond being almost intact $(d=2.56 \AA)$, Fig. 3 (b). Upon further push towards the surface the In adatom splits the dimer bond, overcoming a barrier of $\simeq 0.35 \mathrm{eV}$, and stays shortly within the $Z_{\text {In }}$ channel in a second shallower feature of the PES. The formation of directed In-As bonds (see Fig. 3 (c)) gives rise to a third minimum of $E_{b}$ at $\left(Z_{\text {In }}=1.3, d=5.1 \AA\right)$.
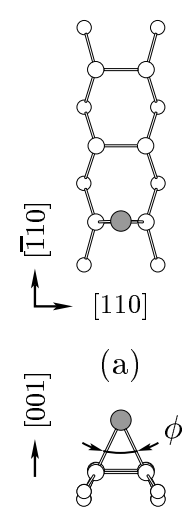

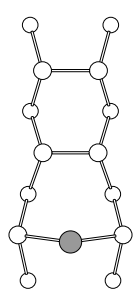

(b)

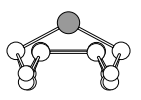

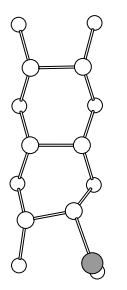

(c)

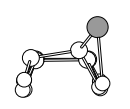

FIG. 4. Different bonding configurations of an In adatom interacting with the edge As dimer; atomic positions in the topmost two atomic layers are shown (In: shaded circle, As: empty circles). (a) In adatom sitting above the closed edge dimer, similar to Fig. 3 (b) (configuration corresponding to the $\mathbf{T}_{3}$ site). $\phi$ indicates the As-In-As bond angle; (b) In adatom splitting the dimer, similar to Fig. 3 (c); (c) In adatom splitting the dimer back-bond.

In a similar way, we analyze the adatom interaction with the edge dimers in the $c(4 \times 4)$ unit cell, Fig. . Since the edge dimer has only one neighbor, the second-layer As atoms are expected to relax more efficiently. Indeed, comparing similar bonding configurations for the In atom at the center dimer, Fig. 3 (c), and at the edge dimer, Fig. 1 (b), we find $E_{b}=-1.3 \mathrm{eV}$ for the latter, which is only slightly lower than $E_{b}\left(\mathbf{T}_{3}\right)$. A third possibility, where In attacks the outer back-bond of an edge dimer, is shown in Fig. 目 (c). This configuration results in $E_{b}=$ $-1.25 \mathrm{eV}$.

It is now clear that additional binding sites for In, related to broken As-As bonds, are energetically higher than adsorption on the PES of Fig. 1, and are therefore not substantially populated in equilibrium. Thus, the mechanism operating in the case of $\mathrm{Ga} / \mathrm{GaAs}(001)$ $\beta 2(2 \times 4)$ is absent in the $\operatorname{In} / \mathrm{GaAs}(001)-c(4 \times 4)$ system Indeed, the more bulky In adatom with an ionic radius $10 R_{\text {In }}$ larger than that of $\mathrm{Ga}$, when inserted into the As dimer, introduces substantial elastic distortion of the dimer As back-bonds that cannot be energetically compensated by the gain due to rehybridization. Note that even in the case of an open dimer the In adatom resides $1.3 \AA$ above it, Fig. 3 (c), which implies an As-In-As bond angle $\phi \simeq 125^{\circ}$, while the Ga adatom is incorporated almost collinearly with the two As atoms, $12 \phi \simeq 175^{\circ}$. In summary, our analysis justifies the use of a single PES (Fig. [1) for In diffusion in the CTRW formalism.

\section{Effect of strain}

The foregoing discussion allows us to single out the main route for the adatom migration: $\mathbf{A}_{1} \stackrel{\mathbf{T}_{1}}{\longleftrightarrow} \mathbf{A}_{1}$. 


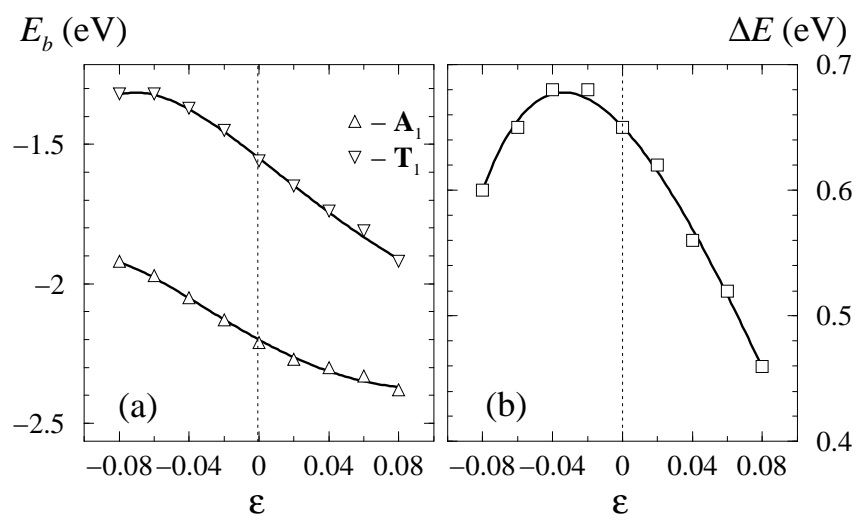

FIG. 5. (a) Binding energy $E_{b}$ as a function of strain $\varepsilon$ for an In adatom at the $\mathbf{A}_{1}$ and $\mathbf{T}_{1}$ sites; (b) diffusion barrier $\Delta E \equiv E_{b}\left(\mathbf{T}_{1}\right)-E_{b}\left(\mathbf{A}_{1}\right)$ as a function of $\varepsilon$. Full curves on both panels represent least-squares polynomial fits to the calculated points.

Hence, the objective in this section is to analyze the influence of surface elastic strain on the $\Gamma_{11}$ rate. A nonvanishing strain field in the substrate results in a different equilibrium configuration of the topmost atomic layers. Consequently, both the surface phonon spectrum and the PES will experience changes affecting in turn both the frequency prefactor and activation energy in the exponential in Eq. (2). The net effect of strain is thus determined by the interplay between the latter two effects. One may expect, however, that the dominant contribution comes from variations in the diffusion barrier $\Delta E \equiv E_{b}\left(\mathbf{T}_{1}\right)-E_{b}\left(\mathbf{A}_{1}\right)$, for it enters an exponential. This motivated us to concentrate mainly on the strain renormalization of $\Delta E$, but our approach allows for the influence of $\Gamma^{(0)}$ to be also incorporated without detailed knowledge of its functional dependence on strain.

For each particular value of $\varepsilon$ the In adatom is placed above the $\mathbf{A}_{1}$ and $\mathbf{T}_{1}$ sites and the same relaxation scheme as for mapping the PES, Sec. IIIA, is applied to obtain the respective binding energy $E_{b}(\varepsilon)$. The calculated values are shown in Fig. 5. Interestingly, we find that $\Delta E(\varepsilon)$ is a monotonically decreasing function for any tensile strain $(\varepsilon>0)$ employed in the calculations, Fig. ( (b). To be specific, this behavior has its onset at $\simeq 3 \%$ compressive strain, where $\Delta E$ reaches a maximum of $0.68 \mathrm{eV}$. Applying larger compressive strain leads to a reduction of $\Delta E$, with the $\Delta E(0)$ value recovered again for $\varepsilon=-0.06$. The non-monotonic dependence on strain can be rationalized by inspecting the $E_{b}(\varepsilon)$ curves, given in Fig. 5 (a). While for $\varepsilon<0 E_{b}$ at the adsorption site $\mathbf{A}_{1}$ follows a linear law with a slope of $-3.8 \mathrm{eV}$, the binding energy at the saddle point $\mathbf{T}_{1}$ contains, although small, non-linear terms in strain which do not cancel in the evaluation of $\Delta E$. For an inhomogeneously strained sample, the pronounced strain dependence of $E_{b}$ for both the adsorption site and the saddle point will introduce a position dependence of $\Delta E$. This finding complies with none of the two limiting scenarios of changes of $\Delta E$ discussed in the literature 19 where only either $E_{b}\left(\mathbf{A}_{i}\right)$ or $E_{b}\left(\mathbf{T}_{k}\right)$ contributes. We would also like to emphasize that the commonly employed linearity 8 for the strain dependence of the diffusion barrier $\delta(\Delta E(\varepsilon))$ is not justified in the case of $\mathrm{In} / \mathrm{GaAs}(001)-c(4 \times 4)$ as clearly seen from Fig. 5 (b). Thus one needs to go to higher order terms in $\varepsilon$ to adequately describe the observed $\delta(\Delta E(\varepsilon))$ behavior. It is also important to point out that strain does not change qualitatively the discussion about the interaction of the In adatom with As-As bonds, based on the PES in Fig. 3. Extensive tests over the entire range of strain considered here were carried out for $E_{b}$ of In at the two stable minima, Fig. 3 (b) and (c). We found that the binding configuration of Fig. 3 (b) was always slightly preferable over the one in Fig. 3 (c), but the strongest binding site for In remains to be $\mathbf{A}_{1}$.

Up to now, the reports in the literature about the effect of strain on the diffusion barrier are scarce. A slight lowering of the diffusion barrier upon tensile strain $(\varepsilon>3.5 \%)$ has been reported for Ag self-diffusion on a $\mathrm{Ag}(111)$ slab within the effective-medium theory. 12 The first-principles treatment of the same system 18 has found instead a linear increase of the barrier with strain. For semiconductors, a lowering of the diffusion barrier upon tensile strain has only been remorted for $\mathrm{Si}$ adatom and dimer diffusion on $\mathrm{Si}(001), 2123$ although the underlying binding trends inferred from the MD simulation $\$ 21$ are opposite to those shown in Fig. 同 (a).

Given the strain dependence of the diffusion barrier described above, the basic question arises whether diffusion limitations can be observed in the growth kinetics of InAs on GaAs. This would be the case if the adparticle diffusivity is reduced for relevant material parameters and growth conditions.

As the substrate around an InAs island, e.g. of pyramidal or truncated pyramidal shape, is under compressive strain, cf. Ref. 15 and Fig. 8 below, an indium adatom approaching the island samples the $\varepsilon<0$ branch of $\Delta E(\varepsilon)$. This branch is accurately described by

$$
\delta(\Delta E(\varepsilon))=\delta E_{\max }\left[1-\left(\frac{\varepsilon}{\left|\varepsilon_{\max }\right|}+1\right)^{2}\right], \quad \varepsilon<0 .
$$

Eq. (5) gives the excess diffusion barrier over the one for the unstrained surface $\Delta E(0)$, parameterized by the maximum excess $\delta E_{\max }=30 \mathrm{meV}$, and the strain value at which it occurs, $\varepsilon_{\max }=-3 \%$. On the basis of Eq. (5), one can write a rather general expression for the diffusion coefficient taking account of the effect of strain,

$$
D(\varepsilon)=D_{0}(1+2 \varepsilon) \delta \Gamma^{(0)}(\varepsilon) \exp \left[-\frac{\delta(\Delta E(\varepsilon))}{k_{\mathrm{B}} T}\right],
$$

where $D_{0} \equiv$ const is the value of $D$ for the unstrained surface, $\delta \Gamma^{(0)}=\Gamma^{(0)}(\varepsilon) / \Gamma^{(0)}(0)$ is the reduction or enhancement factor of the attempt frequency $\Gamma^{(0)}$, and $2 \varepsilon \equiv \operatorname{Tr} \varepsilon_{\alpha \beta}$ is the relative change in the surface area. 


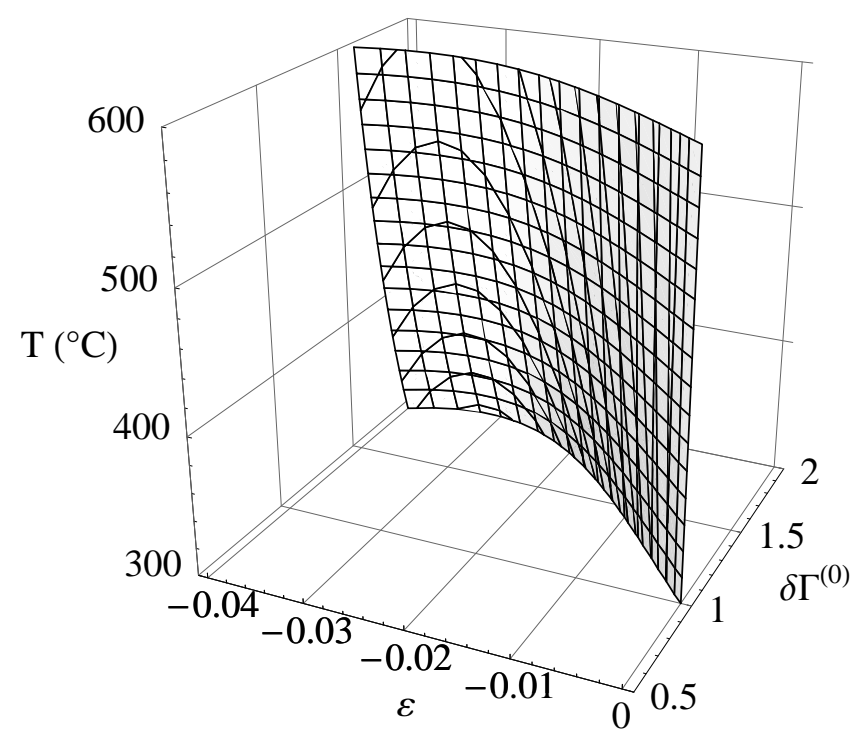

FIG. 6. The $D / D_{0}=1$ isosurface in the $3 \mathrm{D}$ parameter space $\left(\varepsilon, \delta \Gamma^{(0)}, T\right)$. The view point is from the side where $D / D_{0}<1$. Points beyond the isosurface correspond to enhanced diffusivity $D / D_{0}>1$.

A first estimate of the expected reduction of $D$ within the typical temperature range $350-500^{\circ} \mathrm{C}$ used for InAs deposition on the $c(4 \times 4)$-reconstructed $\mathrm{GaAs}(001)$ substrate 30 can be obtained by inserting Eq. (5) in (6), setting $\delta \Gamma^{(0)} \equiv 1$. The resulting reduction, $D\left(\varepsilon_{\max }\right) / D_{0} \simeq$ 0.6 , turns out to be small due to the smallness of $\delta E_{\max }$. As a consequence, changes of the prefactor due to the effect of strain on lattice vibrations are equally important in determining the strain renormalization of the In diffusivity on the $\operatorname{GaAs}(001)-c(4 \times 4)$ surface in the relevant temperature regime.

Although it is possible to obtain $\delta \Gamma^{(0)}$ from firstprinciples calculations, it is difficult to get an estimate that is better than a factor of two with reasonable computational effort. Hence we consider $\delta \Gamma^{(0)}$ as an independent parameter in the following analysis. Thus the right-hand side of Eq. (6) is a function of three parameters, $\varepsilon, \delta \Gamma^{(0)}$, and $T$. The region in parameter space where strain-induced growth limitations can be expected is defined by the requirement

$$
D\left(\varepsilon ; \delta \Gamma^{(0)}, T\right) / D_{0}<1 .
$$

Fig. 6 represents the isosurface $D / D_{0}=1$ in the $3 \mathrm{D}$ parameter space of $\varepsilon, \delta \Gamma^{(0)}$, and $T$. We note that the reduction in diffusivity due to positive $\delta(\Delta E)$, especially for lower temperatures, persists even for $\delta \Gamma^{(0)} \rightarrow 1$, i.e. in presence of the so-called compensation effect 43 However, for a very strong compensation effect, $\delta \Gamma^{(0)}(\varepsilon)>\delta \Gamma_{\mathrm{c}}^{(0)} \simeq$ 2 , no decrease in $D$ in the relevant range of $\varepsilon$ and $T$ values can be expected.

Finally, we have performed DFT calculations to obtain an estimate of $\Gamma_{11}^{(0)}(-0.04)$, using the harmonic approx- imation for the lattice vibrations and a force-constant matrix involving only the degrees of freedom 4 of the In adatom. This estimate indicates $\sim 70 \%$ increase of the prefactor for $4 \%$ compressive strain. In this case one cannot expect more than a few percent maximum reduction of $D$, which would in turn make diffusion limitations for the specific example of the $\operatorname{GaAs}(001)-c(4 \times 4)$ surface rather unlikely.

\section{CONSEQUENCES FOR GROWTH}

It is interesting to discuss the impact of strain on the growth kinetics of both $2 \mathrm{D}$ and $3 \mathrm{D}$ arrays of selfassembled strained islands in a more general context. As already mentioned in the introduction, for these two situations different regimes of strain are realized, and we address them separately.

\section{A. Growth on a capping layer with buried islands}

In analogy to the previous discussion one can also consider diffusion in the regime of tensile strain, $\varepsilon>0$. This situation is pertinent to the heteroepitaxial growth of $3 \mathrm{D}$ arrays of vertically self-organized QDs. When a 2D sheet of QDs is completed and capped by a spacer GaAs layer, the GaAs lattice is expanded in the regions above the buried InAs QDs. We consider the onset of In deposition onto the spacer layer before nucleation of the first new islands. A stationary concentration of adatoms on the surface builds up by the equilibrium between supply from an atomic In beam source and loss due to evaporation of In. However, the concentration may vary laterally along the surface. In the stationary state the local concentration $n\left(\mathbf{r}_{\|}\right)$is given by local equilibrium,

$$
n\left(\mathbf{r}_{\|}\right)=n_{0} \exp \left[-U\left(\mathbf{r}_{\|}\right) / k_{\mathrm{B}} T\right],
$$

where $U\left(\mathbf{r}_{\|}\right)$is the binding energy of the In adatoms at site $\mathbf{A}_{1}$, and $\mathbf{r}_{\|}$is the coordinate within the surface. $U\left(\mathbf{r}_{\|}\right)$is a function of local strain, as given by $E_{b}\left(\varepsilon\left(\mathbf{r}_{\|}\right)\right)$ in Fig. 目 (a). As can be seen from this figure the binding strength increases with increasing strain. Thus, the local concentration of adatoms, and hence the nucleation probability for a new island, is increased in the region above a buried island where the capping layer surface is under tensile strain. Our calculations, thus, provide a microsespic foundation for the frequently made assumption 45 . 1 that it is easier to nucleate an InAs island on those regions of the capping surface where the GaAs lattice constant is widened up and thus more closely matches the InAs lattice constant.

\section{B. Diffusion limitations in island growth kinetics}

The conditions under which kinetic growth limitations can be expected were discussed already in Sec. III (see 


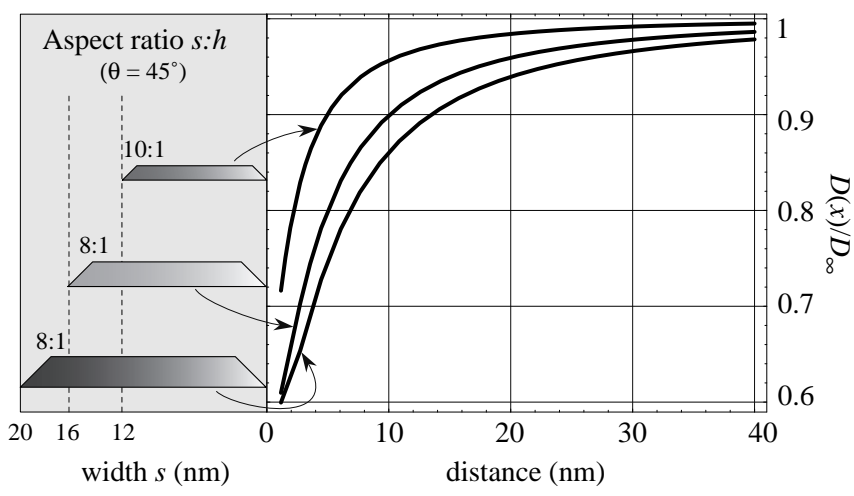

FIG. 7. Strain renormalization of $D(x)$, according to Eqs. (6) and (8), versus island size within the 1D flat island approximation at $T=450^{\circ} \mathrm{C}$ and absence of compensation effect, $\delta \Gamma^{(0)}=1$. $D_{\infty}$ refers to the asymptotic value of $D$ at infinitely large distance from the island. The tilt angle of the island side facets is $\theta$ and $h$ is their height. The island/substrate system is assumed to be InAs/GaAs.

Eq. (7) and Fig. 6). It is interesting to illustrate the possible consequences of such limitations for the island sizes. This is pursued here within the framework of a simple model problem based on the flat island approximation. 17 One might think, for instance, of adatom diffusion towards the extended edge of a quantum wire.

As a first step we address the strain renormalization of the adparticle diffusivity due to an isolated island. The strain field it creates in the underlying substrate surface, within the adopted model, has the form

$$
\varepsilon(x)=\eta \ln \left|\frac{P_{2}(x)}{Q_{2}(x)}\right| .
$$

The island geometry (height $h$, width $s$, and the tilt angle of the side facets $\theta$ ) completely determines the coefficients in the second-order polynomials $P_{2}(x)$ and $Q_{2}(x)$, whereas the elastic properties of the material system (e.g. Poisson ratio, shear modulus of the substrate) enter the prefactor $\eta$.

To assess quantitatively the role of diffusion limitations, we insert the numerical values for $\delta E_{\max }$ and $\varepsilon_{\max }$ obtained in Sec. III C. For three islands of different size Fig. 7 shows the spatial dependence of $D(x)$ obtained by inserting Eq. (8) into Eq. (6), for $\delta \Gamma^{(0)}=1$ and $T=450^{\circ} \mathrm{C}$. As seen from the short-range behavior of the diffusion coefficient, the larger islands can be about 20 $30 \%$ more effective in hindering the adatom migration provided that no substantial compensation effect from the $\Gamma^{(0)}$ prefactor is present. Consequently, as long as the islands grow via strain-dominated surface mass transport and the last mentioned condition is met, the compressive strain field may lead to retarded growth of the larger islands.

Consider now two islands of characteristic size $s_{1}$ and $s_{2}$, with $s_{1}>s_{2}$, separated by a distance $L \gg$ $s_{1}, s_{2}$, Fig. 8. Supply of adparticles to the surface is

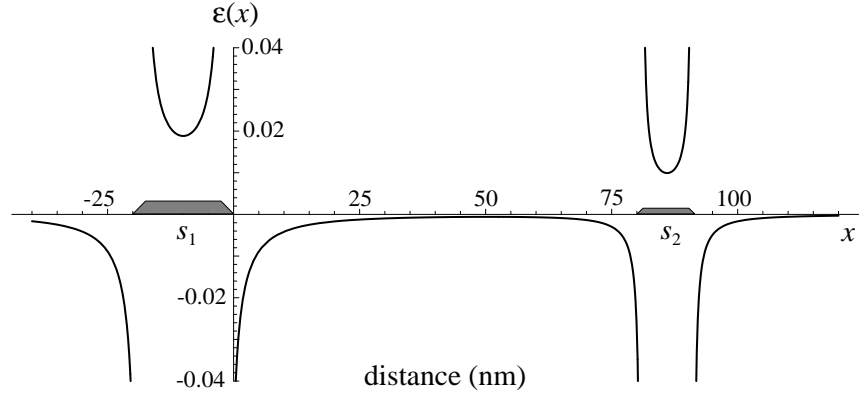

FIG. 8. Strain field $\varepsilon(x)$ at the substrate surface, according to Eq. (8), for two 1D islands of width $s_{1}$ and $s_{2}$ whose edges are separated by distance $L$. The island/substrate system is InAs/GaAs.

maintained by a stationary flux $F_{0}$. One may ask then, what is the steady-state adparticle density distribution $n(x)$ at the surface, and how does it affect the diffusional currents of single adatoms toward the islands $j_{1}$ and $j_{2}$ ? This is a standard problem in kinetics; 48.49 however, we require it to be solved for a spatially varying migration potential $U(x)$ due to the presence of strained islands. Again, we can exploit the results obtained in Sec. III Q, identifying $U(x)$ with $E_{b}(\varepsilon(x))$ for the adsorption site $\mathbf{A}_{1}$. From Fig. 5 (a) it becomes clear that a compressive strain of a few percent significantly weakens the binding of the In adatom at the $\mathbf{A}_{1}$ site. Thus the coherently strained island gives rise to a repulsive potential $U(x)$ that amounts to a few tenths of an $\mathrm{eV}$. The time-dependent single atom-density $n(x, t)$ satisfies a Smoluchowski-type equation50.51 that takes explicit account of the field of force due to $U(x)$,

$$
\frac{\partial n}{\partial t}=\frac{\partial}{\partial x}\left[D(x)\left(\frac{\partial n}{\partial x}+\frac{n}{k_{\mathrm{B}} T} \frac{d U(x)}{d x}\right)\right]+F_{0}
$$

In the simplest case, when the island edges act as perfect sinks, i.e. $n(0)=n(L)=0$, the stationary solution reads as

$$
n(x)=F_{0} e^{-\frac{U(x)}{k_{\mathrm{B}} T}} \int_{0}^{x} \frac{x_{0}-x^{\prime}}{D\left(x^{\prime}\right)} e^{\frac{U\left(x^{\prime}\right)}{k_{\mathrm{B}} T}} d x^{\prime},
$$

with $0<x_{0}<L$ being the position between the two islands where the total adparticle current vanishes $j\left(x_{0}\right) \propto$ $-\left.\nabla n\right|_{x_{0}}=0$

$$
x_{0}=\left[\int_{0}^{L} \frac{1}{\tilde{D}(x)} d x\right]_{0}^{-1} \int_{0}^{L} \frac{x}{\tilde{D}(x)} d x,
$$

with $\tilde{D}(x)=D(x) \exp \left[-U(x) / k_{\mathrm{B}} T\right]$. It is now straightforward to obtain the result that relates $j_{1}$ with $j_{2}$

$$
\left|\frac{j_{1}}{j_{2}}\right|=\frac{x_{0}}{L-x_{0}} .
$$


Without the effect of strain, the adatom density has a simple parabolic profile,

$$
n_{0}(x)=\frac{F_{0}}{2 D_{0}}\left(2 x_{0}-x\right) x
$$

with its maximum being exactly at the midpoint between the two islands, $x_{0}=L / 2$. The strain renormalization of diffusion shifts $x_{0}$ towards the bigger island, thus reducing the particle current reaching this island. This simple 1D model problem demonstrates that the smaller island will grow faster, until $x_{0}$ gets shifted back towards the midpoint when the sizes of the two islands have become equal. As a consequence, the strain-limited adatom diffusion will tend to equalize the island sizes by controlling the capture areas for the two islands competing for the deposited material. We note, however, that this effect will be reduced if a compensation from the frequency prefactor $\Gamma^{(0)}$ is operative.

\section{SUMMARY AND DISCUSSION}

We have presented the first $a b$ initio analysis of the effect of strain on adatom diffusivity in the context of the heteroepitaxial growth of InAs QDs on GaAs(001). In particular, we quantified the strain dependence of the diffusion barrier for an indium adatom on the GaAs(001)$c(4 \times 4)$ surface. The In interaction with the surface As dimers was also given due account. A simple 1D model problem was employed to demonstrate that the strainlimited diffusion contains an archetype of self-limiting growth of strained islands. We note that the self-limiting effect is an intrinsic feature for a system with spatially varying diffusion coefficients, as indicated by Eqs. (11) and (12), bearing reduction within a certain operative range of strain values. For the lattice-mismatched heteroepitaxy of semiconductor nanostructures, the strain effect will give rise to a significant repulsive interaction between a strained island and an adatom diffusing towards the island. Moreover, the diffusivity will be reduced as well, but a very accurate treatment is needed to assess its reduction. Our atomistic calculations yielded a maximum increase of the In diffusion barrier on $\mathrm{GaAs}(001)-c(4 \times 4)$ of $30 \mathrm{meV}$ for compressive misfit strain. Since this value is of the order of $k_{\mathrm{B}} T$, conclusions about the relevance of this effect would require as well an accurate calculation of the prefactor $\Gamma^{(0)}$, which is beyond our present goal.

Finally, we would like to comment on other possible extensions of the work presented here. The discussion presented in this article refers to diffusivity of a single adatom, the so-called tracer diffusion coefficient 25 (sometimes denoted in the literature as $D^{*}$ ). Our microscopic results could, however, serve as an input, for example, to Monte Carlo simulations in order to shed more light on the effect of strain on adatom diffusivity. Experimentally, the self-assembled coherent islands are usually grown on a wetting layer, and thus the In adatoms, in fact, diffuse on an InAs wetting layer or on one of mixed (In,Ga)As composition. Therefore the $\mathrm{In} / \operatorname{GaAs}(001)-c(4 \times 4)$ system used here is suitable for modeling the arrival of the first In atom to the GaAs surface in the initial stages of InAs deposition, but the results cannot be taken over directly to the later stages of island growth. Future research will therefore have to consider diffusion on a wetting layer and possible account of alloying.52

\section{ACKNOWLEDGMENTS}

One of the authors (E. P.) is much indebted to Prof. T. Mishonov for the fruitful discussions, his suggestions and help during this study. We thank also Prof. E. Schöll, S. Bose and M. Meixner for extensive discussions. This work was partially supported by Deutsche Forschungsgemeinschaft (Sfb 296).

${ }^{1}$ D. J. Eaglesham and M. Cerullo, Phys. Rev. Lett. 64, 1943 (1990); Y.-W. Mo, D. E. Savage, B. S. Swartzentruber, and M. G. Lagally, Phys. Rev. Lett. 65, 1020 (1990); J. M. Moison, F. Houzay, L. Leprince, E. André, and O. Vatel, Appl. Phys. Lett. 64, 196 (1994).

${ }^{2}$ D. Leonard, K. Pond, and P. M. Petroff, Phys. Rev. B 50, 11687 (1994).

${ }^{3}$ N. N. Ledentsov, V. M. Ustinov, V. A. Shchukin, P. S. Kop'ev, Zh. I. Alferov, and D. Bimberg, Semiconductors 32, 343 (1998).

${ }^{4}$ C. Priester and M. Lannoo, Phys. Rev. Lett. 75, 93 (1995).

${ }^{5}$ V. A. Shchukin, N. N. Ledentsov, P. S. Kop'ev, and D. Bimberg, Phys. Rev. Lett. 75, 2968 (1995); V. A. Shchukin and D. Bimberg, Rev. Mod. Phys. 71, 1125 (1999) and references therein.

${ }^{6}$ L. G. Wang, P. Kratzer, M. Scheffler, and N. Moll, Phys. Rev. Lett. 82, 4042 (1999); L. G. Wang, P. Kratzer, N. Moll, and M. Scheffler, Phys. Rev. B 62, 1897 (2000).

${ }^{7}$ H. T. Dobbs, D. D. Vvedensky, A. Zangwill, J. Johanson, N. Carlsson, and W. Seifert, Phys. Rev. Lett. 79, 897 (1997);

${ }^{8}$ H. T. Dobbs, A. Zangwill, and D. D. Vvedensky, in Surface Diffusion: Atomistic and Collective Processes, edited by M. Tringides (Plenum Press, New York, 1997).

${ }^{9}$ H. M. Koduvely and A. Zangwill, Phys. Rev. B 60, R2204 (1999);

${ }^{10}$ Y. Chen and J. Washburn, Phys. Rev. Lett. 77, 4046 (1996).

${ }^{11}$ D. E. Jesson, G. Chen, K. M. Chen, and S. J. Pennycook, Phys. Rev. Lett. 80, 5156 (1998).

12 A. Kley, P. Ruggerone, and M. Scheffler, Phys. Rev. Lett. 79, 5278 (1997); A. Kley, Theoretische Untersuchungen zur Adatomdiffusion auf niederindizierten Oberflächen von GaAs (Wissenschaft \& Technik Verlag, Berlin, 1997). 
${ }^{13}$ J. G. LePage, M. Alouani, D. L. Dorsey, J. W. Wilkins, and P. E. Blöchl, Phys. Rev. B 58, 1499 (1998).

${ }^{14}$ S. V. Ghaisas, Surf. Sci. 223, 441 (1989); see also S. V. Ghaisas and A. Madhukar, J. Vac. Sci. Technol. B 7, 264 (1989).

${ }^{15}$ N. Moll, M. Scheffler, and E. Pehlke, Phys. Rev. B 58, 4566 (1998).

${ }^{16}$ Q. Xie, A. Madhukar, P. Chen, and N. Kobayashi, Phys. Rev. Lett. 75, 2542 (1995).

17 O. G. Schmidt and K. Eberl, Phys. Rev. B 61, 13721 (2000).

18 C. Ratsch, A. P. Seitsonen, and M. Scheffler, Phys. Rev. B 55, 6750 (1997).

19 M. Schroeder and D. E. Wolf, Surf. Sci. 375, 129 (1997); see also J. M. Cohen, Surf. Sci. Lett. 306, L545 (1994); R. Ferrando and G. Tréglia, Phys. Rev. Lett. 76, 2109 (1996).

${ }^{20}$ C. C. Matthai and G. A. Moran, Appl. Phys. Lett. 123/124, 653 (1998).

${ }^{21}$ C. Roland and G. H. Gilmer, Phys. Rev. B 46, 13428 (1992).

${ }^{22}$ H. Spjut and D. A. Faux, Surf. Sci. 306, 233 (1994).

${ }^{23}$ E. Zoethout, O. Gürlü, H. J. W. Zandvliet, and B. Poelsema, Surf. Sci. 452, 247 (2000).

24 J. Philibert, Atom movements: diffusion and mass transport in solids (Les Edition de Physique, Les Ulis, 1991).

${ }^{25}$ R. Gomer, Rep. Prog. Phys. 53, 917 (1990).

${ }^{26}$ D. J. Srolovitz, Acta Metall. 37, 621 (1989).

${ }^{27}$ Q. Xie, P. Chen, and A. Madhukar, Appl. Phys. Lett. 65, 2051 (1994).

28 T. R. Ramachandran, R. Heitz, P. Chen, and A. Madhukar, Appl. Phys. Lett. 70, 640 (1997)

${ }^{29}$ M. Sauvage-Simkin, R. Pinchaux, J. Massies, P. Calvarie, N. Jedrecy, J. Bonnet, and I. K. Robinson, Phys. Rev. Lett. 62, 563 (1989); D. K. Biegelsen, R. D. Bringans, J. E. Northrup, and L.-E. Swartz, Phys. Rev. B 41, 5701 (1990).

${ }^{30}$ B. A. Joyce, D. D. Vvedensky, A. R. Avery, J. G. Belk, H. T. Dobbs, and T. S. Jones, Appl. Surf. Sci. 130-132, 357 (1998).

${ }^{31}$ L. G. Wang, P. Kratzer, and M. Scheffler, Jpn. J. Appl. Phys. 39, Pt. 1, No. 7B, 4298 (2000).

${ }^{32} \mathrm{P}$. Ruggerone, C. Ratsch, and M. Scheffler, in The chemical Physics of Solid Surfaces 8, edited by D. A. King and D. P. Woodruff (Elsevier, Amsterdam, 1997), and references therein.

${ }^{33}$ M. Bockstedte, A. Kley, J. Neugebauer, and M. Scheffler, Comp. Phys. Commun. 107, 187 (1997); http://www.fhi-berlin.mpg.de/th/fhimd/.

${ }^{34}$ M. Fuchs and M. Scheffler, Comp. Phys. Commun. 119, 67 (1999).

35 J. P. Perdew, K. Burke, and M. Ernzerhof, Phys. Rev. Lett. 77, 3865 (1996).

${ }^{36}$ Account of the spin polarization within GGA lowers the energy of the free In atom by $\Delta E_{\mathrm{s}}=0.16 \mathrm{eV}$; M. Fuchs, private communication.

37 G.-M. Rignanese, Ph. Ghosez, J.-C. Charlier, J.-P. Michenaud, and X. Gonze, Phys. Rev. B 52, 8160 (1995).

${ }^{38}$ P. Hänggi, P. Talkner, and M. Borkovec, Rev. Mod. Phys. 62, 251 (1990).

39 J. W. Haus and K. W. Kehr, Phys. Rep. 150, 264 (1987), and references therein.
${ }^{40}$ D. R. Lide, editor, Handbook of Chemistry and Physics (CRC Press, NY, 1995); for example, at four-fold coordination $R_{\mathrm{In}^{3+}} / R_{\mathrm{Ga}^{3+}} \simeq 1.3$.

${ }^{41}$ F. Bechstedt and R. Enderlein, Semiconductor surfaces and interfaces (Akademie-Verlag, Berlin, 1988), Table 4.2.

${ }^{42}$ H. Brune, K. Bromann, H. Röder, K. Kern, J. Jacobsen, P. Stolze, K. Jacobsen, and J. Nørskov, Phys. Rev. B 52, R14 380 (1995).

${ }^{43}$ G. Boisvert, L. J. Lewis, and A. Yelon, Phys. Rev. Lett. 75, 469 (1995)

${ }^{44}$ C. Ratsch and M. Scheffler, Phys. Rev. B 58, 13163 (1998).

${ }^{45}$ J. Tersoff, C. Teichert, and M. G. Lagally, Phys. Rev. Lett. 76, 1675 (1996), and references therein.

${ }^{46}$ V. Holý, G. Springholz, M. Pinczolits, and G. Bauer, Phys. Rev. Lett. 83, 356 (1999).

47 J. Tersoff and R. M. Tromp, Phys. Rev. Lett. 70, 2782 (1993).

${ }^{48}$ W. K. Burton, N. Cabrera, and F. Frank, Phil. Trans. Roy. Soc. 243, 299 (1951).

${ }^{49}$ J. Tersoff, M. D. Johnson, and B. G. Orr, Phys. Rev. Lett. 78, 282 (1997).

${ }^{50}$ R. Festa and E. Galleani d'Agliano, Physica A 90, 245 (1978).

${ }^{51}$ H. Risken, The Fokker-Planck Equation (Springer, Berlin, 1996).

${ }^{52}$ E. Penev, P. Kratzer, and M. Scheffler, in preparation. 